\title{
Mémoires en relation directe avec les problèmes d'équipement
}

\author{
PAR M. KAMMERLOCHER

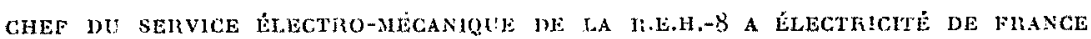

Parmi les mémoires présentés au Congrès de Montréal en relation directe avec des problèmes d'équipement, nous avons reten'u la presque totalité de ceux qui concernent l'hydraulique des vanmes faisant lobjet du sujel $A$, ainsi que certains mémoires traitant des problèmes d'équipement d'écluses, sujet $B$, ou des entraînements d'air, sujet C.

\section{HYDRAULIQUE DES VANNES (Sujet A)}

Nous pouvons préalablement classer les quelque 40 mémoires présentés sur ce sujet d'une part en un certain nombre de mémoires personnels sur des recherches ou réalisations diverses, et d'autre part en deux ensembles issus, l'un des études faites à propos des vamnes d'Assouan, dont les mémoires ont été présentés conjointement par les ingénieurs égyptiens de « Sadd el Aali Authority » et des ingénieurs de SOGREAH, l'autre des études faites pour des vannes spéciales destinées à des travaux maritimes dont les mémoires ont été présentés par des ingénieurs du Waterloopkundig Laboratorium de Delft.

L'extrême diversité des types de vannes et, dans un même type, la diversité des conceptions technologiques, rendraient souhaitable l'établissement d'une doctrine générale permettant d'appliquer à chaque cas la solution la mieux adaptée.

Il n'a pas été présenté de synthèse d'ensemble sur ces problemes, mais seulement des syntheses partielles, en particulier sur les deux principaux sujets suivants :

- D'une part l'incidence de la conception d'une vanne sur les efforts auxquels elle est soumise et sur les risques de vibrations et de cavilation;

.... D'autre part, les recherches sur la réaction du matériel soumis à des efrorts hydrodynamiques fluctuants, la prédiction des dangers de vibrations el leurs conséquences sur la tenue du matériel.

L'ensemble constitué pax ces travaux, par certaines études complémentaires et la description d'installations ou de matériels correspondant à des expériences industrielles intéressantes, apporte une contrihution utile à l'établissement d'une future doctrine générale.

\section{Efforts de manceuvre :}

Une première catégorie d'études peul être classée sous la rubrique: «Déterminalion des efforts de manouvre et de leurs fluctuations $\gg$. Ce sont principalement les mémoires suivants :

$\mathrm{N}^{\circ} 5 \mathrm{~A}$ : «Forces hydrauliques sur une vanne levante verticale », par le professeur-docteur A. GRzYwIENsKr.

N 104: « Recherches sur la force hydrodynamique fluctuante agissant sur une vanne de fond et sa représentation sur modèle », par A.S. ABrecrv.

N" $16 \mathrm{~A}$ : « Facteurs agissant sur les forces de manouvre d'une vanne verticale », par le Dr M.A. SFim el J. Duport. 
$\mathrm{N}^{\circ} 24 \mathrm{~A}$ : « Forces tangentielles exercées par l'écoulement et par les vagues sur les vannes-secteurs en ouverture partielle $»$, par M. Dirkzwager el J.E. Prins.

$\mathrm{N}^{\circ} 30 \mathrm{~A}$ : «Dynamique d’une vanne à glissement et d'un barrage mobile soumis à des vibrations et anx forces exercées par des vagues », par H.S. SchoEmaker.

Des communications de M. Grzywienski, Selim-Duport, Dirkzwager-Prins, on peut dégager cerlains éléments de doctrine en ce qui concerne linfluence de la position du couteau, du bordé de la vanne et de la disposition des élanchéités, sur les efforts fluctuants ou non, auxquels se trouvent soumis les organes de manceuve d'une vanne.

M. Abelev, dans son mémoire, apporte une contribution importante aux doctrines de recherches, en démontrant que dans une bande très étendue du nombre de Reynolds (d'environ 3000 à 80000 ), les forces hydrodynamiques fluctuantes, agissant sur une vanne plate, peuvent être reproduites sur le modèle de la même façon que le sont les forces permanentes. On peut s'attendre à ce qu'il en soit ainsi a fortiori pour les nombres de Reynolds plus élevés que 80000 et on peut, à notre avis, s'attendre aussi à ce que le même résultat s'applique à toutes les forces hydrodynamiques fluctuantes ayant leur origine dans un décollement franc de la veine liquide. De son côté, M. Schoemaker a fait la synthèse des travaux du groupe hollandais et complète les conclusions de $M$. Abelev en montrant la possibilité de réaliser des modèles qui permettent de reproduire pratiquement les mouvements de la structure sous l'effet des forces fluctuantes.

Nous signalons en passant que cette méthode avait été appliquée à la recherche des efforts sur les tourillons des ćléments papillons réglables de la vanne installée dans l'aménagement marémoteur expérimental de Saint-Malo.

\section{Conception des grandes vannes plates :}

On peut classer sous cette deuxième rubrique les recherches sur les éléments essentiels des grandes vannes plates. Ce sont: les études sur Ia position relative du couteau, la conception des étanchéités et du bordé, les formes et les dispositions des rainures. Ces trois sujets sont abordés dans les mémoires $n^{08} 5 \mathrm{~A}$ et $10 \mathrm{~A}$ du professeur Grzywienski et de M. Abelev déjà cités, ainsi que dans les mémoires du groupe ingénieurs égyptiens-SOGREAH, et dans certains mémoires du groupe hollandais.

En ce qui concerne la disposition du couteau et celle de l'étanchéité par rapport au bordé de la vanne, il ne se dégage pas de conclusion générale évidente, et nous avons noté qu'aucun des auteurs n'a mentionné l'influence très grande que peuvent avoir ces deux dispositions sur les limites de cavitation.

L'apport concret de ces différents mémoires se trouve:

- A nouveau dans les conclusions du mémoire de M. Abelev, démontrant que les forces hydrodynamiques fluctuantes peuvent être reproduites convenablement sur le modèle, comme cela a été dit pour les efforts de manouvre.

- Dans les conclusions des mémoires de MM. Abelev et Duport-Bertrand montrant que les trois types de fonctionnement des vannes plates en conduite, c'est-à-dire : avec jet noyé, avec jet libre on en ressaut noyé, se présentent de façon bien différente, aussi bien du point de vue des efforts hydrodynamiques que des limites de cavitation.

- Dans une analyse assez complète présentie par les mémoires du groupe égyptienSOGREAH, de l'origine des vortex et de la cavitation dans les rainures de vannes plates.

Cette dernière analyse montre, en particulier, l'intérêt qu'il peut $\mathrm{y}$ avoir à décaler les rainures suffisamment à l'aval du plan du bordé et du couteau. L'un des mémoires cités décrit des essais effectués avec un type nouveau de vanne conçu à partir de cette idée directrice.

\section{Forces fluctuantes, vagues et vibrations :}

II est également intéressant de grouper sous cette troisième rubrique celles des communicaions qui traitent de l'action des pressions fluctuantes, et des vagues ainsi que de l'étude des vibrations.

Ces sujets sont abordés dans les rapports suivants :

N" $2 \mathrm{~A}$ : « Batardage par une boule sphérique d'une conduite débitant à gueule bée », par M. Gérodolle.

$\mathrm{N}^{\circ} 5 \mathrm{~A}$ : «Forces hydrauliques sur une vanne levante verticale », par Je professeur-docteur A. Grzywlenskr.

$\mathrm{N}^{\circ} 11 \mathrm{~A}$ : « Vanne à éléments papillons horizontaux d'orientation réglable », par MM. KammerLochen et DAuMr.

No $14 \mathrm{~A}$ : «Etudes hydrauliques de vannes et de puits pour un tunnel de dérivation », par M. S. ANeElin et dans le groupe des mémoires hollandais.

S'il se dégage peu de points de doctrine géné- 
rale au point de vue des conceptions constructives, on trouve du moins à ce sujet des indications intéressantes, par exemple:

- Sur l'influence de la postion du couteau (communication $\mathrm{n}^{\circ} 24 \mathrm{~A}$ par M. Dirkzwager et J.E. PRINS).

- Sur l'influence de l'inclinaison du bordé au point de vue des gifles dues aux vagues (communication $n^{\circ} 25$ de MM. Aartsen, E.W. BígKer et W.C. Bischoff VAN HeEmskerck).

Par contre, les doctrines concernant les procédés de recherche et d'interprétation d'essais, sont assez largement traitées, et on peut tirer quelques conclusions très importantes, d'ailleurs déjà mentionnées au moins en partie dans l'analyse des deux rubriques précédentes.

La première conclusion est qu'un modèle réduit peut reproduire correctement les forces hydrodynamiques fluctuantes auxquelles une vanne est soumise.

L'autre conclusion concerne les procédés qui peuvent être envisagés pour l'étude de la réaction d'une vanne à ces efforts fluctuants. Elle peut être formulée par la traduction d'une partie des conclusions du mémoire de M. Schoemaker :

«Des modèles de structure réalisés à l'échelle pour ce qui concerne les propriétés dynamiques (rigidité et répartition des masses solides), donnent un moyen de traiter les problèmes de forces variant rapidement et au hasard.

"En raison du fait que les matériaux disponibles possédant un module d'élasticité suffisamment bas, présentent également une dissipation interne d'énergie élevée, l'utilisation de cette méthode est sérieusement limitée.

« Si des morceaux importants de la structure se déplacent pratiquement en bloc, la réponse de la structure à des forces fluctuantes peut être reproduite dans un modèle qui n'est déformable qu'en certains points particuliers. En ces points, la souplesse peut être reproduite à l'échelle au moyen de pièces métalliques ayant peu d'amortissement interne.

《 Cette méthode est indispensable quand des vibrations auto-entretenues sont à redouter, et quand l'amortissement dû à l'eau est important par rapport aux forces d'excitation.

"Dans le cas où les grandeurs qui déterminent le comportement de la structure ne sont pas liées par une influence mutuelle, la méthode classique qui consiste à mesurer directement ces grandeurs peut être appliquée. »

Ces conclusions de M. Schoemaker résument les données de la technologie des essais sur la détermination des réactions aux efforts fluc- tuants, qui s'apliqueraient aussi bien aux modèles réduits de turbines hydrauliques. Nous y voyons, pour le laboratoire, la possibilité d'apporter au bureau d'études du constructeur et au maitre d'œuvre des informations plus complètes que celles communément demandées au modèle réduit.

Comme il s'agit d'une des conclusions de portẻe générale les plus importantes, tirées des travaux de ce Congrès, nous jugeons utile d'en préciser l'essentiel comme suit.

Dans le cas où l'on redoute une influence mutuelle de la vibration mécanique sur l'écoulement, il est nécessaire que les mouvements du modèle soient reproduits à l'échelle. Si l'ensemble de la structure étudiée possède dans toutes ses parties une souplesse ou une possibilité de déformation non négligeable, le modèle sera réalisć entièrement avec un matériau dont le module d'élasticitẻ et la densité devront être à l'échelle. La difficulté pratique tient au fait que les matériaux actuellement connus possèdent un amortissement interne important.

Si l'ensemble de la structure ne comprend que quelques articulations présentant une souplesse caractérisée, on n'aura à tenir compte, pour les pièces essentielles du modèle, que de la similitude de densité et ce n'est qu'aux points d'articulation que l'on aura à reproduire la souplesse au moyen de pièces métalliques.

Dans le cas où il n'y a pas d'interaction à prévoir entre les mouvements de la structure et l'écoulement, il suffira d'enregistrer les pressions et les forces fluctuantes sans chercher à reproduire les mouvements de la structure qui peuvent être étudiẻs, soit par le calcul, soit sur des modèles de structure indépendants du modèle hydraulique, à partir des efforts enregistrés sur ce dernier.

\section{Expériences industrielles intéressantes :}

Parmi les mémoires traitant plus spécialement d'étude ou de réalisation de vannes d'un type nouveau, nous citerons :

- Les mémoires $7 \mathrm{~A}$ du professeur B. HellStrom, et $15 \mathrm{~A}$, du $\mathrm{D}^{\mathrm{r}} \mathrm{K}$. Prtrikat, présentant chacun une application différente de vannes secteurs s'èclipsant vers le bas.

- Le mémoire de W. Thomas sur une vanne plate à jet circulaire en conduite, avec dispositif particulier d'aération du jet, utilisée aux U.S.A., notamment à Shasta Dam.

- Les deux mémoires $11 \mathrm{~A}$ et $12 \mathrm{~A}$ que nous avons présentés en coopération avec des ingénieurs de Bouchayer-Vtallet et SOGREAH. Le premier sur la vanne de garde de $10 \mathrm{~m}$ 
de hauteur à 3 papillons d'orientation réglable, de $3,50 \mathrm{~m} \times 12 \mathrm{~m}$ de largeur, prévue pour $350 \mathrm{~m}^{3} / \mathrm{s}$, actuellement en fonctionnement dans l'aménagement marémoteur expérimental de Saint-Malo. Le second sur les essais de vannes de fond à papillons verticaux jumelés, prévus pour $400 \mathrm{~m}^{3} / \mathrm{s}$ sous $1 \mathrm{~m}$, étudiés pour une des solutions envisagées dans le projet de l'usine de la Rance.

Ces papillons jumelés présentaient un double intérèt technologique. D’une part, leur forme hélicoildale permeltait de réduire à $48 \%$ le couple maximum sur les tourillons. D'autre part, leur jumelage permettait d'opposer les efrorts des servo-moteurs et d'annuler ainsi leur transmission au béton d'ancrage.

- Le mémoire $14 \mathrm{~A}$ de $\mathrm{S}$. Angelin sur la conception d'un evacuateur en puits dont l'étude apporte une contribution intéressante à la recherche de l'incidence des fluctuations cie pression sur les limites de la cavitation.

Il faut, enfin, faire ćlat de façon particulière des travaux présentés par l'équipe hollandaise du Waterloopkundig Laboratorium sux les éludes failes à propos de vannes spéciales.
Il s'agit d'abord, pour linstallation du Haringvliet, d'un ensemble de deux vannes-secteurs opposées, à double effet, avec pivot hors d'cau au niveau +11 et seuil au niveau $-5,50$, de $56,50 \mathrm{~m}$ de largeur, de $10,50 \mathrm{~m}$ de hauteur et d'un rayon unitaire de $26,50 \mathrm{~m}$.

Ces vannes ont fait l'objet de recherches en laboratoire, notamment sur l'effet de vague (gilles), les vibrations en position noyée, et l'influence de certains paramètres sur les efforts hydrodynamiques, tels l'inclinaison du tablier sur le seuil, l'effet d'un seuil de dissipation, etc., dont les résultats sont utilisables pour d'autres projets.

Il s'agit ensuite d'une vanne en voûte - ou en visière - d'une portée de $45 \mathrm{~m}$ et pourtant de construction légère, dont les mémoires $27 \mathrm{~A}$ et $29 \mathrm{~A}$ présentent l'étude des étanchéités et des vibrations sous l'effet de forces fluctuantes.

Comme indiqué précédemment, les auteurs ont apporté sur ce dernier point de l'étude des vibrations, une intéressante contribution à la synthèse des recherches sur la similitude du modèle réduit pour la détermination de l'effet des forces variables, notamment l'effet des vagues.

\section{HYDRAULIQUE DES ECLUSES DE NAVIGATION (Sujet B)}

De la vingtaine de mémoires présentés sur ce sujet, intéressant l'équipement des aménagements mixtes (production d'énergie et navigation), nous avons retenu plus particulièrement le mémoire $19 \mathrm{~B}$ « $\mathrm{A}$ propos de quelques grandes écluses de navigation construites en France ces dernières années $»$ par M. P. Lereune, de la SOGREAH.

Cet «A propos» est en fait une synthèse des problèmes posés par les cycles de remplissage et de ridange du sas d'une grande écluse et des dernier's perfectionnements permettant l'accélération de ces opérations. II apporte une contribution pratique à la détermination du tracé hydraulique des systèmes de remplissage et de vidange afin que ces cycles puissent être effectues rapidement, sans risque pour les bateaux, leurs amarres ou les organes d'équipement. II donne d'intéressants éléments de comparaison entre les solutions appliquées aux écluses de DonzèreMondragon et de Montélimar sur le Rhône, ainsi qu'à celles d'Otmarsheim et de Fessenhein, sur le Rhin.
On peut ensuite raccorder à ce mémoire une série d'études particulières, notamment :

- Le mémoire $18 \mathrm{~B}$ de M. le professeur L. EsCANDE sur \& Le calcul du débit des aqueducs de remplissage des bassins de radouh $\gg$.

- Le mémoire $14 \mathrm{~B}$ du $\mathrm{D}^{\mathrm{r}} \mathrm{K}$. PEtrikat sur «L'effet des vibrations d'une vanne d'écluse ».

- Les deux mémoires $20 \mathrm{~B}$ et $22 \mathrm{~B}$, respectivement de MM. P. Peutot et J. LABÉtovlle de notre Laboratoire National d'Hydraulique, sur « La détermination théorique et graphique des efforts d'amarrage et l'influence de divers paramètres sur ces efforts pendant le remplissage d'un sas d'écluse. 》

Parmi les idées nouvelles, nous avons retenu celle, pour le moins originale, présentée par lc mémoire $1 \mathrm{~B}$ du professeur $W$. Jarockr, sur la recherche de l'économie d'eau par l'utilisation de deux sas et la mise en cuvre d'un dispositif pneumatique soit sur des sas flotlants soit sous des fonds à niveau variables. 


\section{ENTRAINEMENT D'AIR ET VENTOUSES D'AERATION (Sujet D)}

17 mémoires ont été présentés sous cette rubrique. Nous en avons retenu 6 qui sont en relation directe avec des problèmes d'équipement, dont 3 traitent des prises d'eau secondaires alimentant des galeries en charge dans des installations hydroélectriques.

Le mémoire $17 \mathrm{D}$ de M. Cotillow sur « Les adductions dans les galeries en charge et le problème de l'entraînement d'air $»$, apporte non seulement la description de plusieurs dispositions réalisées par Electricité de France :

- dégazage naturel en puits incliné ou non,

- chambre de dégazage,

- dégazeur à dépression maximum,

- adduction par forage avec siphon ou avec vanne automatique,

- conduite à l'intérieur d'un puits, etc.,

mais aussi des éléments de doctrine assez précis et complets sur la facon de choisir entre ces divers types de prises d'eau secondaires.

Le mémoire $10 \mathrm{D}$ de MM. PLichon et Srvin décrit le dispositif particulier désigné sous le nom pittoresque «d'aérateur à casserole »; qui entre d'ailleurs dans une des dispositions décrites par M. Cotillon.

Le mémoire $5 \mathrm{D}$ de $\mathrm{M}$. Bougrton décrit les études de laboratoires conccrnant des siphons et une chambre de désaération à vortex prévue dans une installation australienne des Snowy Mountains. La recherche sur les siphons avait pour ohjet essentiel de limiter l'entrainement d'air au moment de l'amorçage ou du désamorçage. Quant à la chambre à vortex, il nous semble qu'elle constitue un organe de dégazage infiniment plus complexe et probablement nettement plus onéreux que les systèmes présentés par M. Cotillon.

Ces trois mémoires insistent sur le problème du dégazage, et sur des procédés permettant d'éviter les entrainements d'air dans la galerie principale. On sait en effet que c'est là la difficulté la plus ardue à vaincre.

Deux autres mémoires traitent de l'aération par reniflard :

-- Soit derrière une vanne : c'est le sujet du mémoire $11 \mathrm{D}$ de M. LE MÉHaUté sous le titre : «Le calcul des reniflards »;

- Soit dans le coude réunissant un évacuatenr en puits vertical à la galerie d'exacuation :

\author{
c'est le problème abordé par M. Martins \\ dans le mémoire $4 \mathrm{D}$.
}

M. Le Méha'uté traite d'une façon assez synthétique les deux questions suivantes: Quand faut-il disposer un reniflard à l'aval d'une vanne? Quelle dimension doit-on lui donner? On retrouve dans son exposé la description des divers modes de fonctionnement possibles d'une vanne, ce qui rejoint certains points des mémoires de MM. ABELEv et Dupont sur les vannes.

Doit-on prévoir un reniflard pour aérer la partie convexe du coude au pied d'un ćvacualeur en puits?

- Où ce reniflard doit-il exactement déboucher dans la galerie?

- Quelles dimensions doit-il avoir?

Ce sont là les deux problèmes abordés par M. Martins, qui nous apporte ici le fruit d'études faites au Portugal pour les barrages de Montargil, de Maranao et de Paradela.

Enfin, bien que l'appareil qu'ils décrivent ne soit pas a priori destiné aux équipements hydroćlectriques, mais à celui des égouts d'une grande cité, nous croyons devoir mentionner la vanne automatique très simple et très rustique présentée par MM. Hausser et Derachenal dans leur mémoire $3 \mathrm{D}$ : " Vanne régulatrice pour égout, réglée par entraînement d'air ».

\section{$*$}

Je voudrais maintenant signaler deux manifestations relatires aux sous-commissions spécialisées de l'A.I.R.H. Il s'agit :

- D'une part,, de la mise à l'étude, par le Comité directeur de l'A.I.R.H., de la création d'une Sous-Commission « Vannes et Robinets » à la suite de la demande faite en séance par M. Robert Bouchayer;

- D'autre part, de la décision prise par la SousCommission « Machines Hydrauliques el Cavitation $»$, présidée par le professeur Daily du Massachirsetts Institute, d'élargir les bases d'activité de l'A.I.R.H. dans le domaine des turbines hydrauliques et de la cavitation, en organisant, entre deux congrès généraux, un congris régional reserve aux recherches sur les turbines hydrauliques, restant entendu que, ce résullat ctant alleint, des sessions sur 
ces sujets seraient organisées a'u cours des congrès généraux de l'Association.

Sur ce dernier point, deux invitations ont été examinées: l'une du professeur Numachi, de l'Université de Tokyo, qui désirait organiser ce petit congrès à l'occasion de l'inauguration, dans son laboratoire, de nouvelles installations de recherches sur la cavitation; l'autre, d'origine franco-italienne, présentée par M. Danel.

L'éloignement du Japon et le fait que beaucoup de chercheurs hydrauliciens du Nouveau Monde et d'Europe ne sont pas membres de l'A.I.R.H. ont fait retenir la proposition présentée par M. Danel.

Ce petit congrès, pour lequel de fructueux contacts ont été pris avec notre société, se tiendrait en septembre 1960 , les séances pouvant être partagées entre Nice et San Remo.

Dans cette perspective et pour terminer, qu'il me soit permis de souhaiter à ces prochaines journées techniques des discussions plus animées qu'à Montréal, où les mémoires ont été distribués trop tardivement pour en permettre l'étude préalable approfondie.

L'importance du sujet qui, pour limité qu'il soit, est en création continue, le patronage de notre Société, ses méthodes et son expérience en la matière, devraient assurer l'intérêt technique de ce prochain carrefour.

\section{DISCUSSION}

(Président : M. Chapouthier)

M. le Président remercie M. Kammerlocher et invite les assistants à présenter leur observations.

M. Dupont revient sur ce que M. Kammencochen a dit en évoquant les études hollandaises sur les vibrations des vannes et l'influence des efforts fluctuants : à son avis, ces méthodes, qui ont été présentées de façon très synthétique par M. Scrozmaker, ne peuvent pas être considérées comme nouvelles, car elles étaient déjà implicitement dans les lois de similitude comnues depuis longtemps; mais il lui semble qu'elles contiennent des renseignements assez précis sur la validité de ces lois de similitude et sur les méthodes employées pour les mettre en ouvre.

M. le Président se pose le problème des relations entre deux similitudes, celle de l'hydraulique et celle de la théorie de l'élasticité. Il demande à M. Kolsiman, qui est, comme le rappelle M. Duport, l'auteur d'une des plus intéressantes communications sur ce sujet, si le modèle peut reproduire a la fois un phénomène hydraulique et ses rẻpereussions mécaniques sur un ouvrage métallique.

M. Kolisman répond que, comme le suppose M. le Prèsident, la technique qu'il a exposểe est nouvelle en ce qui concerne l'adaptation de la conformité du modèle à la conformité de l'eat. Le premier article au sujet des modèles en plastique a déjà été publié en 1950 (Proceedings of the Society for Experimental Stress Anatyses, vol. XI, $\mathrm{n}^{\circ} 2$, p. 81 . - G. O. SANKEY : Plastic models for vibration analysis).

II $y$ a une difficulté, parce qu'on ne sait pas exactement si toutes les forces hydrauliques sont reproduites dans le modèle, on s'il y a en ontre une perturbation par suite de cisaillement.

M. Duport remarque qu'en effet il y a, dans ce problème de double représentation hydraulique et structurale, deux sortes de difficultés : celle de faire concorder les échelles de temps, qui est traitée de facon très complète dans les communications de $M$. Kovman et celle de savoir si les efforts hydrodynamiques fluctuants et les vibrations qu'ils exercent sur les vannes sont effectivement reproduits en similitude de Froude, ou bien si certains de ces phénomènes, tels les tourbillons alternés, ne produisent pas des vibrations dont les fréquences propres et mêmes les amplitudes dépendratent du nombre de Reynolds, comme cela a été objecté au cours de l'étude des vannes de Saint-Malo.

Par contre, dans les efforts fluctuants liés à l'écoulcment sous une yanne, la viscosité et le nombre de Reynolds ne semblent pas avoir un effet prépondérant, ce qui rejoint les résultats, d'ailleurs non définitifs, des expériences d'Abelev sur des modèles de vannes.

M. KolKman ajoute que les échelles du modèle qu'il a étudié sont seulement adaptées à l'échelle de Froudc. Pour le barrage type visière, des recherches spéciales ont été faites sur deux modẻles schématisés, afin de déterminer l'influence de l'échelle du modèle sur les vibrations causées par le courant. La dispersion des observations rendait impossible de donner un jugement définitif sur la question. On a l'intention de laire des mesures sur la construction réelle, à peu près terminée, poux comparer avec les données du modèle : fréquences de résonance, amortissement interne de la construction et vibrations excitées par l'eau courante. 